\title{
IDLN-MSP: Idiolocal normalization of real-time methylation-specific PCR for genetic imbalanced DNA specimens
}

\author{
Simeon Santourlidis' ${ }^{1}$ Foued Ghanjati', Agnes Beermann ${ }^{1}$, \\ Thomas Hermanns', and Cédric Poyet ${ }^{2}$ \\ ${ }^{1}$ Institute for Transplantation Diagnostics and Cell Therapeutics, \\ Heinrich Heine University Düsseldorf, Germany and ${ }^{2}$ Department of \\ Urology, University Hospital, University of Zurich, Zurich, Switzerland
}

BioTechniques 60:84-87 (February 2016) doi 10.2144/000114379

Keywords: DNA methylation; methylation-specific PCR; biomarkers; diagnosis; epigenetics

Supplementary material for this article is available at www.BioTechniques.com/article/114379.

Sensitive, accurate, and reliable measurements of tumor cell-specific DNA methylation changes are of fundamental importance in cancer diagnosis, prognosis, and monitoring. Real-time methylation-specific PCR (MSP) using intercalating dyes is an established method of choice for this purpose. Here we present a simple but crucial adaptation of this widely applied method that overcomes a major obstacle: genetic abnormalities in the DNA samples, such as aneuploidy or copy number variations, that could result in inaccurate results due to improper normalization if the copy numbers of the target and reference sequences are not the same. In our idiolocal normalization (IDLN) method, the locus for the normalizing, methylation-independent reference amplification is chosen close to the locus of the methylation-dependent target amplification. This ensures that the copy numbers of both the target and reference sequences will be identical in most cases if they are close enough to each other, resulting in accurate normalization and reliable comparative measurements of DNA methylation in clinical samples when using real-time MSP.

Since its discovery at the beginning of this century (1), many studies have convincingly demonstrated that DNA methylation is of fundamental importance for vertebrate differentiation
(2). It is now evident that a complex and diverse, cell-type specific DNA methylome exists that determines cellular identity and functional states. It constitutes a layer of epigenomic information crucial for gene regulation and cellular differentiation, and it is involved in human disease (3).

The reliable detection of cell-typespecific DNA methylation signatures has become increasingly useful for the identification and genome integrity control of various stem cell types (e.g., induced pluripotent stem cells and mesenchymal stem cells), which are broadly used for research and certain clinical applications such as cell replacement therapies. In cancer, many DNA methylation biomarkers with likely diagnostic, prognostic, and predictive power are in clinical trials, particularly because tumor-derived, aberrantly methylated circulating DNA can be detected in the plasma and other body fluids of cancer patients (4). Few of these biomarkers, however, are presently used in clinical settings $(5,6)$.

In this context, methylation-specific PCR (MSP) (7) is generally accepted as an analytically suitable technique for accurately interrogating DNA methylation at single $\mathrm{CpG}$ sites. DNA methylation detection can be carried out in a precise, semi-quantitative manner by using intercalating dyes (e.g., SYBR Green) in real-time MSP, which provides high analytical sensitivity and a further improvement in specificity by decreasing the numbers of falsepositive and false-negative cases in cancer diagnosis (8). For these reasons this method has already been used in clinical applications for the diagnosis of cancer (9).

In this method the following formula is used:

$\Delta \Delta \mathrm{C}_{\mathrm{q}}=\Delta \mathrm{C}_{\mathrm{q}}\left(\mathrm{C}_{\mathrm{q}}\right.$, sample (target locus) - $\mathrm{C}_{\mathrm{q}}$, sample (reference)) - $\Delta \mathrm{C}_{\mathrm{q}}\left(\mathrm{C}_{\mathrm{q}}\right.$, calibrator (target locus) - $\mathrm{C}_{q}$, calibrator (reference)).

For the $\Delta \Delta \mathrm{C}_{\mathrm{q}}$ calculation to be valid and to result in reliable measurements, the amplification efficiencies of the methylation-dependent target and of the methylation-independent reference must be approximately equal (10), and it is presumed that the copy numbers

\section{METHOD SUMMARY}

In our idiolocal normalization (IDLN) modification of real-time methylation-specific PCR (MSP) assay, the methylationindependent reference sequence used for normalization is selected to be very close to the methylation-dependent target sequence. This guarantees that the copy numbers of the reference and target sequences will be equal in samples such as tumor DNA that may have copy number variations affecting either the reference or target sequence, which could lead to incorrect real-time MSP results. 
of these loci are identical in all samples. Unfortunately, this latter prerequisite is in reality compromised by the numerous genetic abnormalities that are prevalent even during early cancer development (11) for almost every cancer type (12) as well as in pluripotent stem cells in culture (13). That means that application of this method to tumor cell DNA harbors the serious danger of false-negative and false-positive detection due to unequal numbers of targeted DNA loci within the compared samples. Unfortunately, it is not feasible to determine copy number variations for each patient DNA sample before applying MSP.

This serious problem was recognized and described previously for MethylLight assays and has been tackled by the significant improvement of using Alu sequences, depleted of $\mathrm{CpG}$ dinucleotides, in the control reactions (14). Later on, this kind of normalization has been adopted for SMART-PCR (8) since it minimizes normalization errors caused by aneuploidy and copy number changes often observed in cancer cells (15).

Here we describe a simple technical adaptation of intercalating dye real-time MSP, which we have named idiolocal normalization (IDLN), that overcomes this problem and broadens the application of the method for diagnostic purposes. We chose the locus for reference amplification to be close to the locus of target amplification. Thus, the copy numbers of both the reference and target template sequences for a $\Delta \mathrm{C}_{\mathrm{q}}$ measurement should be equal in almost every case, guaranteeing that the measurements will be comparable. Genetic changes (e.g., translocations, deletions, etc.) will only interfere with the results in the rare cases where they have affected the short segment between the reference and target template sequences. Therefore, the closer the reference sequence lies to target sequence, the better.

Theoretical models easily demonstrate how aneuploidy at the involved target loci that changes the $\mathrm{C}_{\mathrm{q}}$ values inserted into the $2^{-\Delta \Delta C_{q}}$ formula inevitably results in a misleading DNA methylation value. For instance, the occurrence of a triploid target locus for the methylationdependent target amplification would lead to an overestimate of methylation.

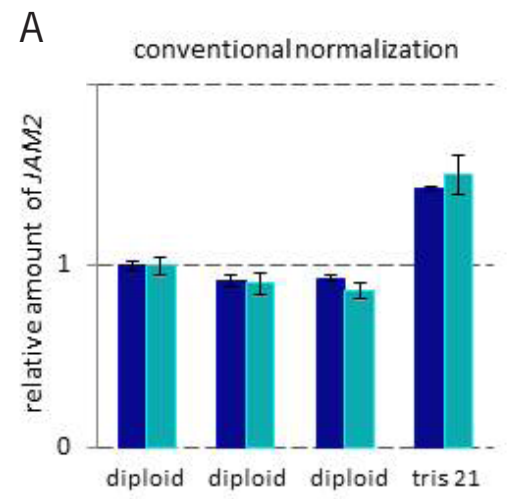

Figure 1. SYBR Green real-time PCR on two JAM2 (Chr 21) gene segments in diploid and trisomic 21 (tris 21) DNA samples. Real-time PCR was been performed on two adjacent JAM2 gene segments (chr 21) with diploid DNA and DNA with trisomy 21. One set of real-time PCR data was conventionally normalized by reference amplification of an NKP46 gene segment (chr 19) (A), while the other was normalized by idiolocal normalization (IDLN) with a reference JAM2 gene segment near the target JAM2 gene segment (B). Target JAM2a gene segment bars are dark blue; Target $J A M 2 b$ gene segment bars are light blue.

$B$
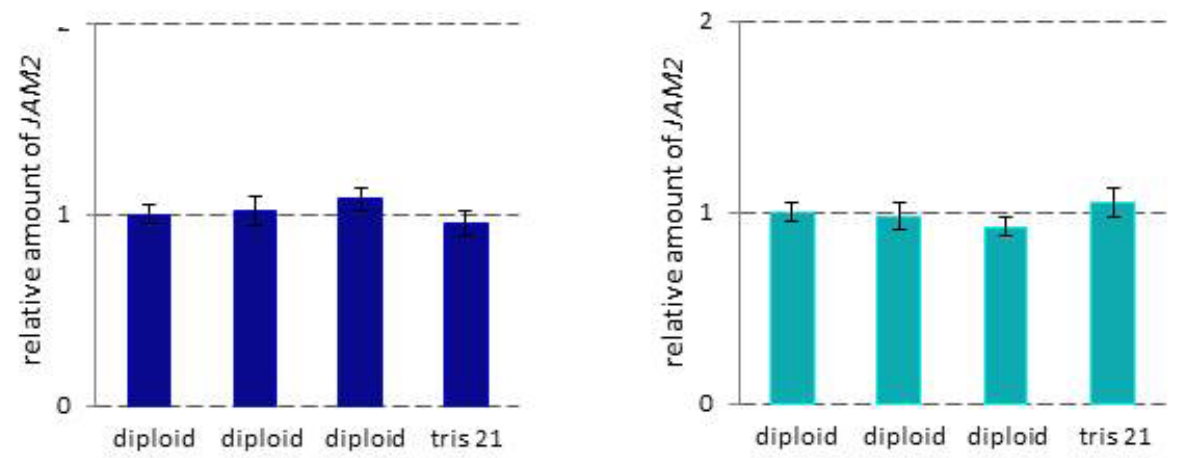

To test if this is really valid in practice, we first chose an experimental set-up mimicking a triploid situation (Figure 1). In this example, we measured by SYBR Green real-time PCR the amount of 2 JAM2 gene segments (JAM2a and JAM2b) that are $136 \mathrm{bp}$ apart from each other on chromosome 21 in equal amounts of DNA samples from diploid individuals and probands with trisomy 21. For each JAM2 gene segment, we either normalized conventionally with the unlinked diploid gene locus NKp46 on chromosome 19 or by our IDLN method using the other JAM2 gene segment as the reference for normalization (see protocol in the Supplementary Material for details). With IDLN, the same relative amount of each JAM2 target segment on chromosome 21 was repeatedly measured for the trisomic as well as the disomic DNA samples (Figure 1B). In contrast, the conventionally normalized MSP approach using an unlinked reference locus that was disomic in all samples clearly indicated an enrichment of the JAM2 gene segments within the trisomic DNA sample (Figure 1A).

This result suggests that methylation of an unaltered, fully methylated target locus would be overestimated by SYBR Green MSP in a trisomic situation if normalization was conventionally conducted using an arbitrarily chosen disomic normalization locus. On the other hand, correct levels of DNA methylation would be measured in a trisomic situation by IDLN since it compensates for underlying genetic imbalances within samples so that methylation-dependent amplifications remain solely dependent on the methylation status.

It would also be expected that an arbitrarily chosen normalization locus that is present in higher or lower copy numbers, such as commonly occurs in tumor DNA samples, would also result in false DNA methylation values. To test this, we first precisely determined the representative methylation status of LINE-1 elements in urinary cell DNA of one bladder cancer patient and one healthy proband. LINE-1 hypomethylation in bladder cancer has been described as a common, early, and, for diagnostic purposes, very promising DNA methylation alteration (16). The results of our bisulfite genomic sequencing reveal the relative DNA methylation of every single CpG dinucleotide within the relevant, distinct 
TAKE ACTION AGAINST CONTAMINATION WITH $\mathrm{QMI}^{\oplus}$ SAFE-SEPTUM

Sample, Inoculate Or Add Nutrients To Your Bioreactor Aseptically !

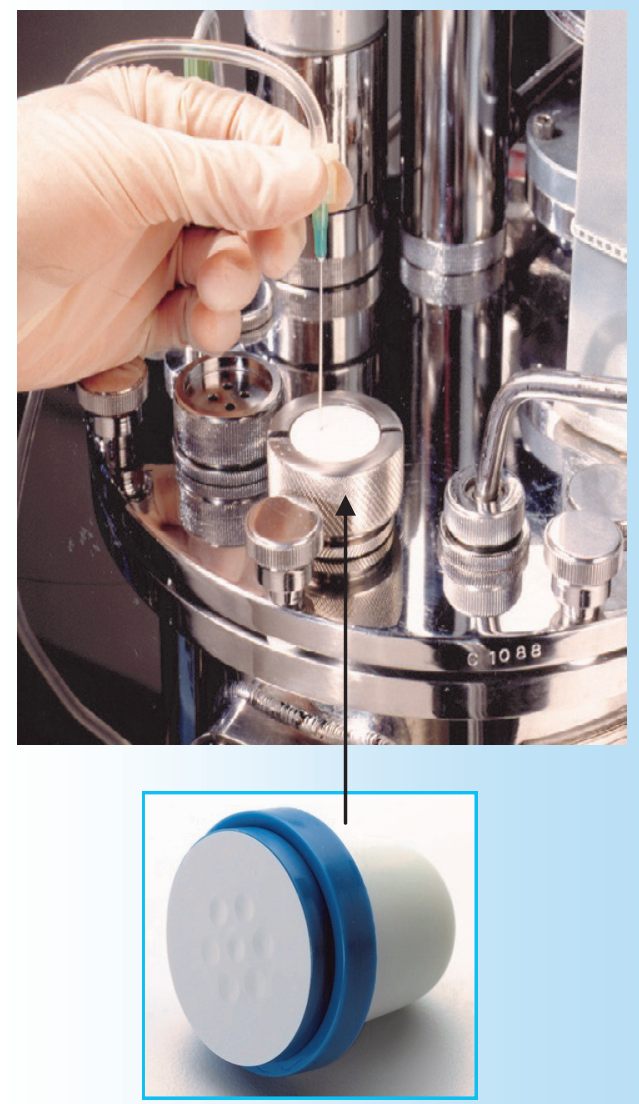

The QMI Safe-Septum is:

- Aseptic

- Pressure \& Temperature Safe

- Pre-Sterilized

- Easy To Retrofit

- Validated

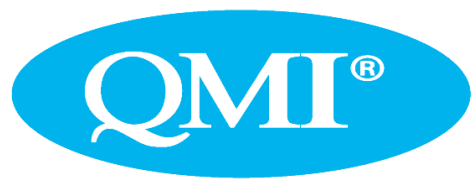

651-501-2337

Email: info@qmisystems.com

A

ZH 16 urinary cellular DNA

ZH 317 urinary cellular DNA

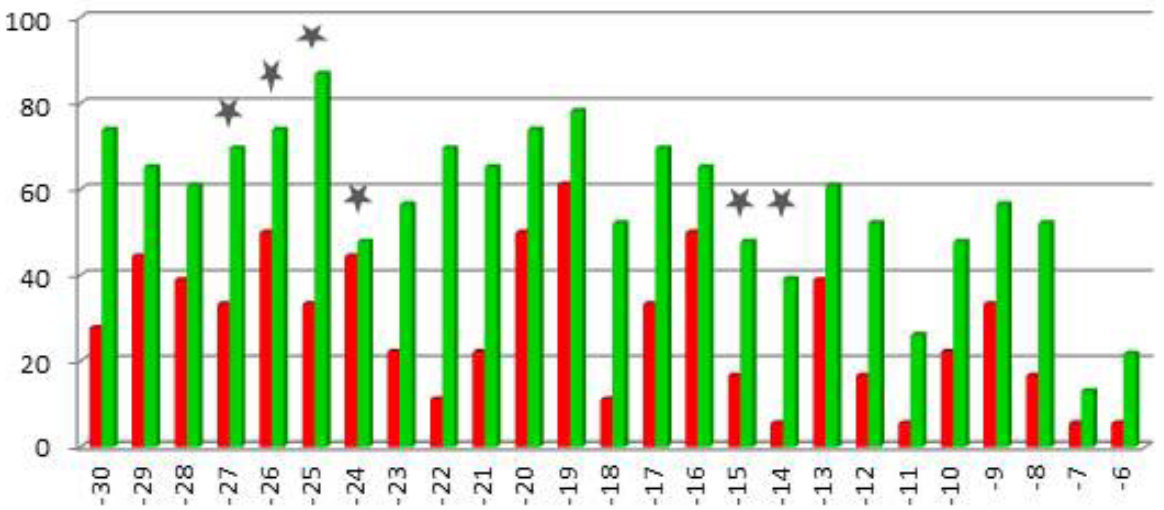

CPG-position upstream of LINE-1 open reading frame 1, relative to ATG start codon

B
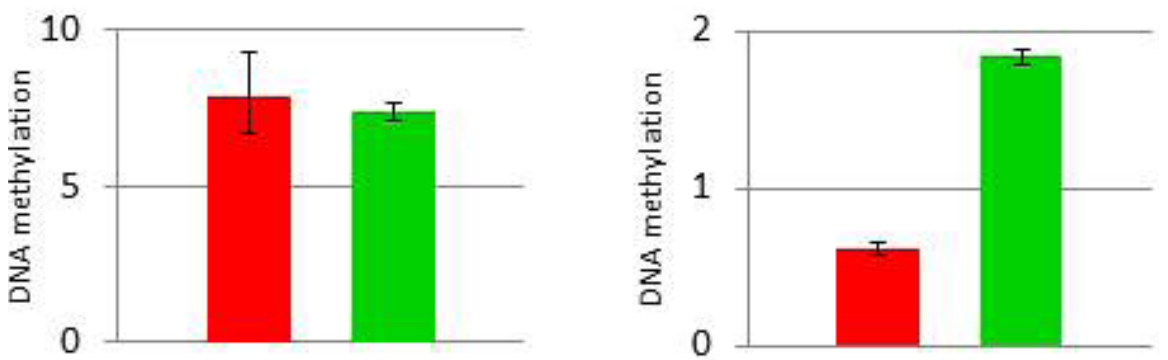

Figure 2. Bisulfite sequencing, conventional normalized real-time methylation-specific PCR (MSP), and idiolocal normalization (IDLN)-MSP of urinary cellular DNA from one bladder cancer patient and one healthy proband. DNA methylation profiles of 436-bp long, CpG-rich, LINE-1 promoter regions from urinary cellular DNA of one bladder cancer patient ( $\mathrm{ZH} \mathrm{16}$, red) and one healthy proband ( $\mathrm{ZH}$ 317, green) were analyzed by bisulfite genomic sequencing as shown in (A). Each bar shows the frequency of methylated $\mathrm{CpG}$ at the $\mathrm{CpG}$ position indicated by number below the bar. $\mathrm{CpG}$ positions are relative to the ATG start codon of first $L I N E-1$ open reading frame; for example, - 6 means the sixth CpG upstream of this ATG. Stars above the bars indicate the positions of the primers used in the real-time MSP assays on the same samples. This result is shown in (B), where the left panel shows the GAPDH-normalized result and the right panel shows the idiolocal normalization result.

promoter segment of LINE-1 DNA elements (Figure 2A). Only real-time MSP using IDLN with a reference sequence $185 \mathrm{bp}$ downstream of the target sequence in the LINE-1 promoter (see protocol in the Supplementary Material for details) reproduced the results from bisulfite sequencing showing hypomethylation of bladder cancer patient DNA, whereas the real-time MSP result normalized conventionally with an unlinked gene locus (GAPDH) yields a misleading result (Figure $2 \mathrm{~B}$ ) due to the aneuploidy found in transitional cell carcinoma of the bladder (17). The reliability of IDLN real-time MSP has been validated for this experimental set-up in additional patient samples. In addition, we have also validated the procedure by direct comparison to conventionally normalized measure- ments in more than 100 samples of cell-free and cellular urinary DNA (4).

Therefore, for all measurements using relative quantifying intercalating dye real-time MSP in DNA samples with likely genetic imbalances, idiolocal normalization of real-time MSP is a useful option. Samples of this sort include not only cancer samples but also cells after prolonged cultivation, (e.g., stem cells).

\section{Author contributions}

S.S. contributed to the conception, development, and writing of the manuscript. F.G. contributed to the execution and analysis of experiments. A.B. contributed to the execution and analysis of experiments. T.H. and C.P. defined and prepared clinical samples. 


\section{Acknowledgments}

In Memoriam: Professor Rolf Ackermann. The authors are grateful to Wolfgang Schulz and Nina Graffmann for revising the paper. They thank Barbara Leube for kindly providing trisomy 21 DNA. They thank Janosch Rixius for technical assistance. This study has been supported by: Prof. Dr. Rolf Ackermann, Wissenschaftliche Urologische Gesellschaft e.V. Stiftung für Altersforschung Heinrich-Heine Universität Düsseldorf.

\section{Competing interests}

The authors declare no competing interests.

\section{References}

1. Johnson, T.B. and R.D. Coghill. 1925. The discovery of 5-methyl-cytosine in tuberculinic acid, the nucleic acid of the Tubercle bacillus. J. Am. Chem. Soc. 47:2838-2844.

2. Schübeler, D. 2015. Function and information content of DNA methylation. Nature 517:321-326.

3. Heyn, H. and M. Esteller. 2012. DNA methylation profiling in the clinic: applications and challenges. Nat. Rev. Genet. 13:679-692.

4. Ghanjati, F., A. Beermann, T. Hermanns, C. Poyet, M.J. Araúzo-Bravo, H.H. Seifert, M. Schmidtpeter, W. Goering, et al. 2014. Unreserved application of epigenetic methods to define differences of DNA methylation between urinary cellular and cell-free DNA. Cancer Biomark. 14:295-302.

5. Mikeska, T. and J.M. Craig. 2014. DNA methylation biomarkers: cancer and beyond. Genes (Basel). 5:821-864

6. Payne, S.R. 2010. From discovery to the clinic: The novel DNA methylation biomarker (m)SEPT9 for the detection of colorectal cancer in blood. Epigenomics. 2:575-585.

7. Herman, J.G., J.R. Graff, S. Myöhänen, B.D. Nelkin, and S.B. Baylin. 1996. Methylationspecific PCR: a novel PCR assay for methylation status of $\mathrm{CpG}$ islands. Proc. Natl. Acad. Sci. USA 93:9821-9826.

8. Kristensen, L.S. and L.L. Hansen. 2009. PCR-based methods for detecting singlelocus DNA methylation biomarkers in cancer diagnostics, prognostics, and response to treatment. Clin. Chem. 55:1471-1483.

9. Potter, N.T., P. Hurban, M.N. White, K.D. Whitlock, C.E. Lofton-Day, R. Tetzner, T. Koenig, N.B. Quigley, and G. Weiss. 2014. Validation of a real-time PCR-based qualitative assay for the detection of methylated SEPT9 DNA in human plasma. Clin. Chem. 60:11831191.

10. Livak, K.J. and T.D. Schmittgen. 2001. Analysis of relative gene expression data using real-time quantitative PCR and the $2^{\text {(-Delta Delta } C(T) \text { ). }}$ Method. 25:402-408.

11. Simon, R., H. Bürger, C. Brinkschmidt, W. Böcker, L. Hertle, and H.J. Terpe. 1998.
Chromosomal aberrations associated with invasion in papillary superficial bladder cancer. J. Pathol. 185:345-351.

12. Rabbitts, T.H. 1994. Chromosomal translocations in human cancer. Nature 372:143-149.

13. Peterson, S.E. and J.F. Loring. 2014. Genomic instability in pluripotent stem cells: implications for clinical applications. J. Biol. Chem. 289:45784584.

14. Weisenberger, D.J., M. Campan, T.I. Long, M. Kim, C. Woods, E. Fiala, M. Ehrlich, and P.W. Laird. 2005. Analysis of repetitive element DNA methylation by MethyLight. Nucleic Acids Res. 33:6823-6836.

15. Kristensen, L.S., H.M. Nielsen, H. Hager, and L.L. Hansen. 2011. Methylation of MGMT in malignant pleural mesothelioma occurs in a subset of patients and is associated with the T allele of the rs16906252 MGMT promoter SNP. Lung Cancer 71:130-136.

16. Schulz, W.A., C. Steinhoff, and A.F. Florl. 2006. Methylation of endogenous human retroelements in health and disease. Curr. Top. Microbiol. Immunol. 310:211-250.

17. Fadl-Elmula, I., L. Gorunova, N. Mandahl, P. Elfving, R. Lundgren, F. Mitelman, and S. Heim. 2000. Karyotypic characterization of urinary bladder transitional cell carcinomas. Genes Chromosomes Cancer. 29:256-65.

Received 31 July 2015; accepted 05 October 2015.

Address correspondence to Simeon Santourlidis, Schürkesweg 3, 40670 Meerbusch, Germany. Email: simeon.santourlidis@med.uni-duesseldorf.de

To purchase reprints of this article, contact: biotechniques@fosterprinting.com

\section{ChemiDoc-lt Imager Delivers!}

Western blots DNA

Bacterial Colonies
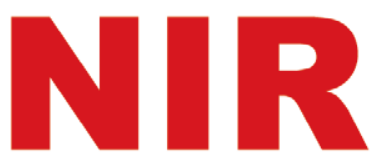

Proteins
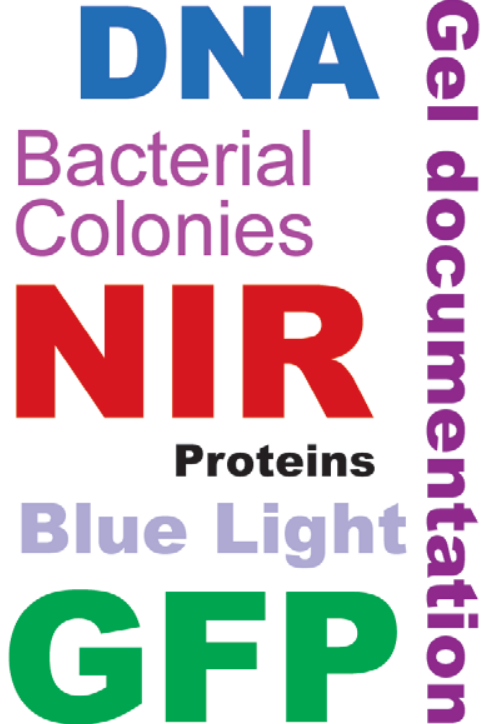

Image Capture \& Analysis

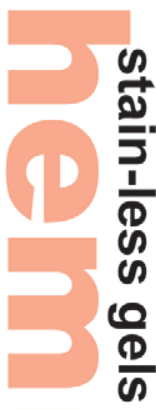

\section{Colorimetric}

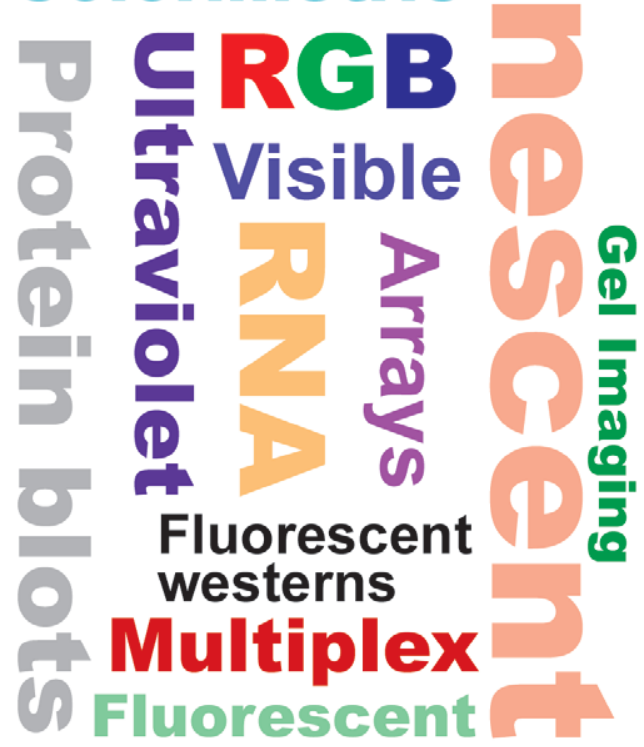

\section{Unlimited Imaging!}

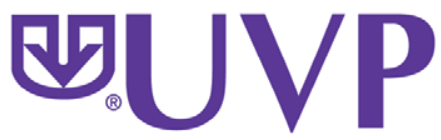

An Analytik Jena Company

UVP, LLC USA: (800) 452-6788 | (909) 946-3197 Cambridge UK: +44(0)1223 420022

uvp.com/ChemiDoc-It2delivers 